\title{
Max-prod eigenvectors and consistency of the preference matrix
}

\author{
Hana TomáškováUniversity of Hradec Králové, Faculty of Informatics and Management, Rokitanského 62, 50003 \\ Hradec Králové, Czech Republice-mail: hana.tomaskova@uhk.cz Martin Gavalec \\ University of Hradec Králové, Faculty of Informatics and Management, Rokitanského 62, 50003 Hradec Králové, \\ Czech Republice-mail: martin.gavalec@uhk.cz
}

\begin{abstract}
Analytical Hierarchy Process can be described as a mathematical model of multi-criteria decision making that uses the decomposition of a complex unstructured situation into simpler parts - the hierarchical system. Using subjective pairwise comparisons the numerical values are assigned to individual components, showing their relative importance. In the paper the preference matrix is preferably processed in the max-prod algebra, which is close to the classical linear algebra. By max-prod algebra we understand a linear structure on a linearly ordered set of real numbers together with the binary operations maximum and multiplication (product), similarly as the addition and multiplication operations are used in the classical linear algebra.

The preference matrix usually is not consistent, so the problem in this paper is formualed as findng the closest consistent approximation of the given preference matrix.
\end{abstract}

Keywords: preference matrix, tropical algebra, AHP decision making, eigenvectors.

JEL classification: C44

AMS classification: $90 \mathrm{C} 15$

The support of the Czech Science Foundation project \# 14-02424S is gratefully acknowledged.

\section{Introduction}

The subjective preferences given by human experts are often inconsistent and do not reflect the deep relations between the processed notions, see [Ramík \& Perzina, 2010]. The standard approach to finding the relative importance vector out of an incosistent matrix uses an eigenvector of the preference matrix computed by the methods of linear algebra, [Saaty, 2003]. Possible use of other non-standard methods in other algebras, such as tropical or fuzzy algebra, is considered in this paper.

Differently from binary operations plus and product in classical linear algebra, the tropical algebra uses binary operation maximum and plus, or maximum and product, or similar combinations with minimum instead of maximum.

Properties of various tropical and fuzzy algebras were studied by many authors, with useful applications. Tropical algebras are important in the study of discrete events systems, the steady states of which correspond to eigenvectors of max-plus or max-prod matrices, see [Butkovič, 2010].

In this paper types of preference matrices are studied by methods used in tropical algebra, in particular in the max-prod algebra. The original preference matrix is processed by max-prod operations, until a steady state is reached. The eigenvector of the matrix then describes the steady state preferences and, therefore, it respects all relations hidden in the original matrix. The optimization problem is to find the closest solution of consistency preference matrix. The types of solutions are descibed by some examples. 
ISAHP Article: Mu, Saaty/A Style Guide for Paper Proposals To Be Submitted to the International Symposium of the Analytic Hierarchy Process 2014, Washington D.C., U.S.A.

\section{Tropical algebras}

Two most frequently used tropical algebras are the max-plus and the max-prod algebra. In this paper we work with preference matrices in the multiplicative form. It is natural, therefore, to process the preference matrix in the max-prod algebra. By max-prod algebra we understand a linear structure on a linearly ordered set $\mathcal{R}$ of real numbers together with the binary operations $\oplus=$ maximum and $\otimes=$ product, similarly as the ordinary addition and multiplication operations are used in the classical linear algebra. The operations $\oplus$ and $\otimes$ are extended to matrices and vectors in a natural way. We should remark that the max-prod algebra is isomorphic to max-plus algebra, with the operations maximum and addition. The eigenvalue of a given max-plus or max-prod matrix and the eigenvectors can be efficiently described by considering cycles in specifically evaluated directed graphs.

\section{Consistent approximation of a given preference matrix}

When the preference matrix suggested by an expert is inconsistent, then the question of finding a consistent approximation of the given expert matrix must be solved - There are various methods used to solve this problem. In this paper the question is considered as an optimization problem, that is, the aim is to find the closest consistent matrix which is as close as possible. To solve the above optimization problem, we have first to describe the set of all possible consistent approximations, and then we have to decide which of them is the closest one, in a suitable metric, to the given inconsistent starting matrix.

The paper is based on the following three facts formulated in the max-prod algebra: 1) any square matrix with 1's on the diagonal is consistent if and only if all matrix columns are co-linear and they all are eigenvectors of the given matrix, 2) every irreducible square matrix has exactly one eigenvalue and at least one eigenvector, which can be computed by standard methods of the max-prod algebra, 3) every reciprocal preference matrix is automatically irreducible by definition, hence the fact 2 is always applicable for all preference matrices.

The optimization method will be described on several examples showing various possible types of the eigenspaces, in which the optimal consistent approximation can be found. Examples of the following cases will be presented

- consistent preference matrix, hence no optimization is necessary

- inconsistent preference matrix and critical cycle meet all edges (only one eigenvector exists in this case and the consistent approximation is unique)

- inconsistent preference matrix and critical cycle meet all edges (more than one eigenvector can exist, hence the consistent approximation is not uniquely determined and the optimization problem can have alternative solutions)

The details of the computation, as well as the suggested optimization metric, will be formulated in the full version of the paper.

\section{References}

[Butkovič, 2010] Butkovič, P. (2010). Max-linear Systems: Theory and Algorithms, Springer Monographs in Mathematics, Springer-Verlag, 2010.

[Mls \& Gavalec, 2009] Mls, K. and Gavalec, M. (2009). Multi-criteria models in autonomous decision making systems. In Proceedings of the 10th International Symposium on the Analytic Hierarchy/Network Process. pp 1-8, University of Pittsburgh, Pittsburgh, Pennsylvania, USA.

[Ramík \& Perzina, 2010] Ramík, J., Perzina, R. (2010). A method for solving fuzzy multicriteria decision problems with dependent criteria, Fuzzy Optimization and Decision Making, Springer Verlag,Vol.9, No.2, 123-141.

[Saaty, 2003] Saaty, T.L. (2003). Decision-making with the AHP: Why is the principal eigenvector necessary, European Journal of Operational Research, Volume 145, Issue 1, 16 February 2003, Pages 85-91. 